\title{
Severity of hypertension as a predictor of initiation of dialysis among study participants with and without diabetes mellitus
}

\author{
Taeko Osawa ${ }^{1}$, Kazuya Fujihara ${ }^{2}$, Mayuko Harada ${ }^{1}$, Masahiko Yamamoto ${ }^{3}$, Masaru \\ Kitazawa $^{1}$, Yasuhiro Matsubayashi ${ }^{1}$, Midori Iwanaga ${ }^{4}$, Takaho Yamada ${ }^{1}$, Hiroyasu Seida ${ }^{5}$, \\ Yoshimi Nakagawa ${ }^{6}$, Hitoshi Shimano ${ }^{6}$, and Hirohito Sone ${ }^{7}$ \\ ${ }^{1}$ Niigata University Faculty of Medicine Graduate School of Medical and Dental Science \\ Department of Hematology Endocrinology and Metabolism \\ ${ }^{2}$ Affiliation not available \\ ${ }^{3}$ Department of Internal Medicine, Niigata University Faculty of Medicine, Niigata, Japan \\ ${ }^{4}$ Department of Internal Medicine, Niigata University Faculty of Medicine, Niigata, Japan \\ ${ }^{5}$ JMDC Inc., Tokyo, Japan \\ ${ }^{6}$ Department of Internal Medicine, University of Tsukuba School of Medicine, Tsukuba, \\ Japan \\ ${ }^{7}$ Niigata University Faculty of Medicine
}

May 13, 2020

\begin{abstract}
Aims: To determine associations between severity of hypertension and risk of starting dialysis in the presence or absence of diabetes mellitus (DM). Methods: A nationwide database with claims data on 258,874 people with and without DM aged 19-72 y in Japan was used to elucidate the impact of severity of hypertension on starting dialysis. Initiation of dialysis was determined from claims using ICD-10 codes and medical procedures. Using multivariate Cox modeling, we investigated severity of hypertension as a predictor of the initiation of dialysis with and without DM. Results: Hypertension was significantly associated with the initiation of dialysis regardless of DM. The incidence of starting dialysis in those with SBP [?]119 mmHg and DM (DM+) was almost the same as in those with SBP [?]150 mmHg and absence of DM (DM-). In comparison with SBP [?]119 $\mathrm{mmHg}$, SBP [?]150 mmHg significantly increased the risk of the initiation of dialysis about 2.5 times regardless of DM+ or DM-. Compared with DM- and SBP [?]119mmHg, the HR for DM+ and SBP [?]150 mmHg was 6.88 (95\% CI 3.66-12.9). Conclusions: Although the risks of hypertension differed only slightly regardless of the presence or absence of DM, risks for the initiation of dialysis with DM+ and SBP [?]119 $\mathrm{mmHg}$ were equivalent to DM- and SBP [?]150 mmHg, indicating more strict blood pressure interventions in DM+ are needed to avoid dialysis. Future studies are needed to clarify the cut-off SBP level to avoid initiation of dialysis considering the risks of strict control of blood pressure.
\end{abstract}

\section{Title}

Severity of hypertension as a predictor of initiation of dialysis among study participants with and without diabetes mellitus

\section{Authors}

Taeko Osawa, $\mathrm{MD}^{1}, \mathrm{PhD}^{1}$, Kazuya Fujihara, $\mathrm{MD}^{1}, \mathrm{PhD}^{1}$, Mayuko Harada Yamada, MD ${ }^{1}$, Masahiko Yamamoto, $\mathrm{MD}^{1}$, Masaru Kitazawa, $\mathrm{MD}^{1}$, Yasuhiro Matsubayashi, $\mathrm{MD}^{1}, \mathrm{PhD}^{1}$, Midori Iwanaga, $\mathrm{MD}^{1}$, Takaho Yamada, $\mathrm{MD}^{1}, \mathrm{PhD}^{1}$,Hiroyasu Seida ${ }^{2}$, Yoshimi Nakagawa, $\mathrm{MD}^{3}, \mathrm{PhD}^{3}$, Hitoshi Shimano, MD ${ }^{3}, \mathrm{PhD}^{3}$, Hirohito Sone, $\mathrm{MD}^{1}, \mathrm{PhD}^{1}, \mathrm{FACP}^{1}$ 
1. Department of Internal Medicine, Niigata University Faculty of Medicine, Niigata, Japan

2. Japan Medical Data Center Co., Ltd., Tokyo, Japan

3. Department of Internal Medicine, University of Tsukuba School of Medicine, Tsukuba, Japan

Running Title: Hypertension and initiation of dialysis

Key words: hypertension, dialysis, persons with and without diabetes

Word count for abstract: 250 words

Word count for main text: 2703 words, 3 tables, 1 Figure

\section{Corresponding Author:}

Kazuya Fujihara, MD, PhD

Niigata University Faculty of Medicine

Department of Internal Medicine

1-757 Asahimachi, Niigata, Niigata, Japan, 951-8510

Phone +81-25-368-9026 Fax +81-29-368-9300

Email kafujihara-dm@umin.ac.jp

\section{Acknowledgements}

This work is supported in part by the Ministry of Health, Labour and Welfare, Japan. No potential conflicts of interest relevant to this article exist. The sponsor had no role in the design and conduct of the study. The authors also thank Mami Haga, Natsuko Tada and Yoko Chino, Niigata University Faculty of Medicine, for excellent secretarial assistance.

Disclosure: There is no risk of disclosure of the identity of all the participants. We could not obtain signed informed consent in all the participants, the opportunity to opt-out was accepted in all the participants.

\section{Abstract:}

Aims: To determine associations between severity of hypertension and risk of starting dialysis in the presence or absence of diabetes mellitus (DM).

Methods: A nationwide database with claims data on 258,874 people with and without DM aged 19-72 y in Japan was used to elucidate the impact of severity of hypertension on starting dialysis. Initiation of dialysis was determined from claims using ICD-10 codes and medical procedures. Using multivariate Cox modeling, we investigated severity of hypertension as a predictor of the initiation of dialysis with and without DM.

Results: Hypertension was significantly associated with the initiation of dialysis regardless of DM. The incidence of starting dialysis in those with SBP [?]119 $\mathrm{mmHg}$ and $\mathrm{DM}(\mathrm{DM}+)$ was almost the same as in those with SBP [?]150 mmHg and absence of DM (DM-). In comparison with SBP [?]119 mmHg, SBP [?]150 $\mathrm{mmHg}$ significantly increased the risk of the initiation of dialysis about 2.5 times regardless of DM+ or DM-. Compared with DM- and SBP [?]119mmHg, the HR for DM+ and SBP [?]150 mmHg was 6.88 (95\% CI 3.66-12.9).

Conclusions: Although the risks of hypertension differed only slightly regardless of the presence or absence of DM, risks for the initiation of dialysis with DM+ and SBP [?]119 $\mathrm{mmHg}$ were equivalent to DM- and SBP [?]150 mmHg, indicating more strict blood pressure interventions in DM+ are needed to avoid dialysis. Future studies are needed to clarify the cut-off SBP level to avoid initiation of dialysis considering the risks of strict control of blood pressure.

*What is already known about this subject? 
1. Since dialysis adversely affects the quality of life and is related to high rates of cardiovascular events and mortality, avoiding the need for dialysis is clinically relevant.

2. Both hyperglycemia and hypertension are highly predictive of kidney disease.

*What does this article add?

1. Compared with DM- and SBP [?]119mmHg, the HR for DM+ and SBP [?]150 mmHg was 6.88 (95\% CI 3.66-12.9).

2. The risks of hypertension were not very different between DM+ and DM-.

3. Risks for the initiation of dialysis with DM+ and SBP [?]119 $\mathrm{mmHg}$ were equivalent to DM- and SBP [?]150 $\mathrm{mmHg}$, indicating stricter blood pressure interventions in $\mathrm{DM}+$ are needed to avoid dialysis.

\begin{abstract}
Abbreviations
BMI Body mass index CKD Chronic kidney disease

CVD Cardiovascular disease
\end{abstract}

DM Diabetes mellitus

eGFR Estimated glomerular filtration rate

ESRD End stage renal disease

GFR glomerular filtration rate

HRs Hazard ratios

SBP Systolic blood pressure

LDL-C Low-density lipoprotein cholesterol

HDL-C High-density lipoprotein cholesterol

\title{
Introduction:
}

Since dialysis adversely affects the quality of life and is related to high rates of cardiovascular events and mortality, avoiding the need for dialysis is clinically relevant. Although both hyperglycemia and hypertension are highly predictive of kidney disease [1], only a few studies have investigated the associations between the severity of hypertension and risk of end-stage renal disease (ESRD) especially the initiation of renal replacement therapy in the presence or absence of diabetes mellitus (DM) in the same cohort at the same time and under the same conditions.

More strict blood pressure targets were recently recommended in the guidelines for hypertension by the American College of Cardiology (ACC) and the American Heart Association (AHA) [2]. In these guidelines, the definition of adult hypertension was reduced from the long-standing threshold of $140 / 90 \mathrm{~mm} \mathrm{Hg}$ to 130/80 $\mathrm{mm} \mathrm{Hg}$. Although DM and hypertension defined as SBP [?]140 $\mathrm{mmHg}$, DBP [?] $90 \mathrm{mmHg}$ or the use of antihypertensive treatment are well-known risk factors for ESRD defined according to the initiation of renal replacement therapy [3], various SBP levels have not been investigated with regard to the prevention of dialysis according to DM status. Such an investigation would have clinical relevance. The risk of chronic kidney disease (CKD) defined as the requirement for dialysis, transplantation or by the notation of kidney disease on the death certificate and confirmed by medical record review significantly increased from SBP [?]160 mmHg compared to SBP ¡120 mmHg with adjustment for DM [4]. Also, the risk of ESRD defined as receipt of renal transplant or maintenance dialysis increased in accordance with increases in SBP with adjustment for DM [5]. Although Hsu et al. [5] investigated the impacts of the presence of DM and stratified SBP on ESRD defined as described above, HbA1c was not used in defining DM. Moreover, only age was adjusted for as a covariate. Tozawa et al. [6] showed that elevated SBP was a risk factor for the development of ESRD among Japanese with and without DM. Also, Iseki et al. [7] showed that hyperglycemia defined as fasting blood glucose [?]126 mmHg was a significant risk factor for the development of ESRD in a Japanese 
general population. However, these studies [6.7] did not use HbA1c to define DM and also did not evaluate the impact of combinations of various SBP cut-offs among people with and without DM on starting dialysis. Thus, the impacts of blood pressure control and cut-off values on renal replacement therapy among people with and without DM are still unknown.

Moreover, although patients with renal disease or on dialysis tend to be prescribed hypertensive medication more often than those without renal disease or on dialysis [1.4.8], these studies [4.5] did not adjust for antihypertensive agents as a covariate. Thus, the effects of antihypertensive medication must be considered in evaluating the impact of various SBP levels on the initiation of dialysis.

Therefore, we investigated the risk of various SBP values for the initiation of dialysis in the presence or absence of DM in addition to considering the risk of various levels of SBP with adjustments for the use of antihypertensive medications.

\section{Materials and Methods}

\section{Study participants}

The present study analyzed data from a nationwide claims-based database that included information on 296,129 people enrolled with a health insurance provider for company employees and their dependents in Japan [9]. Details of the claims data and classifications were described elsewhere [8.9.10]. Patients aged 19-72 years who were followed for at least 3 years from 1 April 2008 to 31 March 2013 were included in this analysis and continued to be followed up to 31 September 2016. We examined data on 296,129 individuals in the present study. We then excluded 37,255 individuals due to missing data and panic data. Finally, this study included 258,874 individuals who were outpatients at the time of baseline measurements $(241,628 \mathrm{DM}$ and 17,246 non-DM).

\section{Definitions}

DM was defined according to the following information obtained from the claims database: fasting plasma glucose (FPG) [?]7.0 mmol/L or HbA1c [?]6.5\% or both in individuals not taking an antidiabetic drug, or use of antidiabetic medication(s) regardless of FPG or HbA1c [10].

Blood pressure was measured at all participating facilities in accordance with the guidelines of the Japanese Society of Hypertension [8]. For medical checkups these guidelines recommended measurement of blood pressure twice by the oscillometric method and averaging the results.

The initiation of dialysis was determined according to claims using medical procedures for the initiation of peritoneal dialysis or hemodialysis after 1 month of follow up.

\section{Statistical analysis}

Categorical variables were expressed as numerals and percentages and were compared with $\chi^{2}$ tests. Continuous variables were expressed as mean $\pm \mathrm{SD}$ and were compared using the unpaired Student'st test or the Mann-Whitney U test based on distribution.

Unadjusted overall time to initiation of dialysis was indicated by Kaplan-Meier analysis with log-rank testing. Cox proportional hazards regression model identified variables related to the initiation of dialysis. Covariates included traditional risk factors for dialysis in each model. Hypertension as a covariate was determined according to SBP diagnosed by seven different cut-offs (i.e., [?]110 $\mathrm{mmHg}$, [?]115 $\mathrm{mmHg}$, [?]120 $\mathrm{mmHg}$, [?]125 mmHg, [?]130 mmHg, [?]140 $\mathrm{mmHg}$, and [?]150 mmHg). Data were compared among 10 groups of participants divided according to combinations of the presence or absence of DM and five stratified levels of SBP (i.e., [?]119 mmHg, 120-129 mmHg, 130-139 mmHg, 140-149 mmHg, and [?]150 mmHg).

Analyses were performed using SPSS (version 19.0, IBM, Chicago, IL, USA). Statistical significance was considered for $\mathrm{P}<0.05$. The Ethics Committee of the Niigata University approved this study.

\section{Results}


Characteristics of individuals with and without dialysis in the presence or absence of DM are shown in Table 1. The medium follow-up period was 5.2 years. During the follow-up, 113 individuals $(0.047 \%)$ in the without DM group ( DM-) and 76 individuals (0.44\%) in the with DM group ( DM+) developed the need for dialysis. The incidence of dialysis was 0.079 per 1000 person-years in the DM- group and 0.672 per 1000 person-years in the DM+ group. As shown in Table 1, among DM-, baseline age, percent of men, body mass index (BMI), smoking rate, SBP, DBP, percent of users of medication for hypertension, and prevalence of coronary artery disease were significantly higher in individuals with dialysis compared with those without dialysis. High-density lipoprotein cholesterol (HDL-C) was significantly lower in individuals with dialysis than without dialysis. Among DM+, baseline percentage of men, SBP, HbA1c, percentages of users of medication for DM and hypertension, and prevalence of coronary artery disease were significantly higher in individuals with dialysis compared with those without dialysis. HDL-C was significantly lower in individuals with dialysis than in those without dialysis.

Table 2 shows Cox proportional hazard models for various risk factors for the initiation of dialysis in participants with and without DM. Each stratified SBP level includes the specified cutoff value and upward (for example, SBP [?]110 $\mathrm{mmHg}$ and upward). SBP [?]140 $\mathrm{mmHg}$ was an independent predictor for the initiation of dialysis in the DM- group whereas SBP [?]150 mmHg was an independent predictor in the DM+ group.

Figure 1 shows the cumulative incidence of the initiation of dialysis according to five stratified SBP values (i.e., [?]119 $\mathrm{mmHg}, 120-129 \mathrm{mmHg}, 130-139 \mathrm{mmHg}, 140-149 \mathrm{mmHg}$, [?]150 $\mathrm{mmHg}$ ) and the presence or absence of DM. Hypertension was an independent predictor of the initiation of dialysis, and the incidence of starting dialysis in the DM+ group with SBP [?]119 $\mathrm{mmHg}$ was almost the same as in the DM- group with SBP [?]150 mmHg.

Table 3 shows Cox proportional hazard models for ten groups divided according to combinations of DM+ and DM- and five stratified levels of systolic SBP (i.e., [?]119 mmHg, 120-129 mmHg, 130-139 mmHg, 140$149 \mathrm{mmHg}$, [?]150 $\mathrm{mmHg}$ ) for the initiation of dialysis. HRs for the initiation of dialysis among DM- and SBP [?]150 $\mathrm{mmHg}$ and DM+ and SBP [?]150 $\mathrm{mmHg}$ were 2.87 (1.55-5.32) and 2.28 (1.03-5.01), respectively, values that were quite similar. Compared with DM- and SBP [?]119 mmHg, HRs for the initiation of dialysis among DM- and SBP [?]150 $\mathrm{mmHg}$, and DM+ and SBP [?]119 $\mathrm{mmHg}$ were about 3 times greater. Compared with DM- and SBP [?]119 $\mathrm{mmHg}$, the HR for the initiation of dialysis in DM+ and SBP [?]150 $\mathrm{mmHg}$ was 6.88 (3.66-12.9). No interaction was observed according to SBP levels and DM status.

\section{Discussion}

This is the first study to elucidate the impact of the severity of hypertension on the initiation of dialysis in people with and without DM in a large-scale longitudinal setting. The risks for the initiation of dialysis in those with $\mathrm{DM}+$ and SBP [?]119 $\mathrm{mmHg}$ were equivalent to those with DM- and SBP [?]150 $\mathrm{mmHg}$, indicating that the presence of DM could indicate the need for more strict blood pressure interventions to avoid dialysis. Also, the risks of hypertension were not very different between those with and without DM. The risk of the initiation of dialysis was almost seven times greater in those with both DM+ and hypertension compared with DM- and non-hypertension. However, we could not use the renal function as a covariate, and SBP was measured at only one point in time. Further studies are needed to confirm our findings considering those important risk factors for the initiation of dialysis.

Recently, more strict blood pressure targets were recommended in accordance with the change in the definition of hypertension from [?]140/90 to [?]130/80 mmHg in the ACC/AHA guidelines. However, the target value for hypertensive individuals to avoid dialysis is still unknown. Although DM and hypertension defined as SBP [?]140 $\mathrm{mmHg}$, DBP [?] $90 \mathrm{mmHg}$ or antihypertensive treatment are well-known risk factors for ESRD defined as initiation of renal replacement therapy [3], no evidence was established for the prevention of dialysis according to DM status. Our findings demonstrated that SBP [?]140 $\mathrm{mmHg}$ was a significant independent predictor for the initiation of dialysis in people without DM, whereas this level increased to SBP [?]150 mmHg in people with DM. However, the risk for the initiation of dialysis for DM+ and, especially, SBP [?]119 mmHg was almost the same as that for DM- and hypertension, especially with SBP [?]150 
mmHg. This indicates that people with DM could need more severe blood pressure interventions to prevent dialysis. However, further studies are needed to confirm our findings including other risk factors such as the duration of hypertension and the eGFR.

Generally, hypertension is a well-known risk factor for renal dysfunction [1]. However, little is known about whether the associations also apply to ESRD, and especially whether such associations also apply to renal replacement therapy, not only ESRD, among people with and without DM. The risk of CKD defined as the requirement for dialysis or transplantation or by the notation of kidney disease on the death certificate and confirmed by medical record review significantly increased from SBP [?]160 mmHg, compared to SBP $¡ 120 \mathrm{mmHg}$ with adjustment for DM [4]. Also, the risk of ESRD defined as receipt of renal transplantation or maintenance dialysis increased along with the SBP level after adjustment for DM [5]. Hypertension is a well-known risk factor for renal dysfunction in patients with DM [11-13], and SBP [?]120 mmHg could be associated with development of nephropathy in patients with DM [14]. SBP reportedly predicts early onset of doubling of serum creatinine concentration or ESRD defined as dialysis or renal transplantation in diabetic patients with nephropathy [15]. Higher SBP increases a risk of ESRD among Japanese people with and without DM [6]. Hyperglycemia defined as fasting blood glucose [?]126 mg/dl $(7.0 \mathrm{mmol} / \mathrm{L})$ is a risk factor for the development of ESRD in a Japanese general population [7]. However, that study did not evaluate the impact of the combination of the SBP cut-offs and the presence or absence of DM on starting dialysis. Hsu et al. [5] showed that all of the stratified SBP values in DM+ had higher impacts on ESRD defined as receipt of renal transplantation or maintenance dialysis than in DM-. These findings are consistent with our results suggesting that elevated SBP is a useful marker for predicting initiation of dialysis as well as DM. However, these studies [4.5] did not adjust for antihypertensive medications as a covariate. Moreover, although HbA1c is the gold standard for reflecting hyperglycemia [16] in clinical settings to evaluate the risk of initiation and development of nephropathy [17-19], that study [5] did not use HbA1c to define DM and adjusted only for age. Moreover, we showed that the risk of initiation of dialysis with DM+, even at SBP [?]119 $\mathrm{mmHg}$, was almost the same as such a risk according to DM- and SBP [?]150 $\mathrm{mmHg}$.

Intensive lowering of SBP increased the risk of eGFR loss with and without DM. In addition, this risk was higher in people with DM [20]. At the same time, strict control of blood pressure increased renal dysfunction due to decreased renal blood flow in patients with DM, especially with progressive atherosclerosis [21]. On the other hand, patients with DM might benefit from intensive lowering of blood pressure regarding CVD risk [22]. Therefore, future studies are needed to conclude the optimal cut-off level of SBP for the initiation of dialysis.

Our present study's strengths were its large sample size and accurate definitions of DM, hypertension, and dialysis based on data from health examinations and a claims database that included information on medical practice, which allowed for the certainty that patients with DM had diabetes and to identify almost all patients who underwent initiation of dialysis during the follow-up.

Our study also had some limitations. First, we could not use the eGFR or proteinuria as a covariate. Unfortunately, serum creatinine level is not always included in medical health checkups in Japan, and there were much missing data on proteinuria. Therefore, further studies are needed to confirm our findings considering those important risk factors for the initiation of dialysis. Second, we were unable to distinguish between type 1 and 2 diabetes. Second, it was also not possible to identify distinction between type 1 and 2 diabetes patients be ascertained. However, type 2 diabetes is more common than type 1 diabetes and accounts for $95 \%$ of diabetes in Japan. Although renal anemia according to the progression of renal failure could affect the HbA1c level, HbA1c was widely used as the glycemic index in clinical practice even among patients with chronic renal failure [23]. Third, we do not include renal transplantation as an endpoint in this study. However, the influence of excluding renal transplantation from the analysis would be minimal because the incidence of renal transplantation is very low in Japan. Fourth, it was not possible to identify participants whose glucose control had either improved or deteriorated during the follow-up period. Also, SBP was measured at only one point in time.

In conclusion, although the risks of hypertension were not very different between DM+ and DM-, the risks for 
the initiation of dialysis in those with DM+ and SBP [?]119 mmHg were equivalent to those with DM- and SBP [?]150 mmHg, indicating that individuals with DM could need more strict blood pressure interventions to avoid dialysis. Future studies are needed to conclude the cut-off level of SBP for the initiation of dialysis under the consideration of the risk of strict control of blood pressure.

Conflict of Interest Statement: none declared

Funding: Japan Society for the Promotion of Science

Contribution statement

T.O. and K.F. developed the study design, researched the data, contributed to discussions, wrote the manuscript, and reviewed and edited the manuscript. H.S. planned and supervised this research, researched the data, contributed to discussions, wrote the manuscript, and reviewed and edited the manuscript. M.H.Y., M.Y., M.K., Y.M., M.I., and T.Y. researched the data, contributed to discussions, wrote the manuscript, and reviewed and edited the manuscript. Y.N. and H.S. researched the data and reviewed and edited the manuscript. H.S. developed the study design, contributed to discussions, and reviewed and edited the manuscript and supervised this research.

Reference

1. Yamagata $K$, Ishida $K$, Sairenchi $T$ et al. Risk factors for chronic kidney disease in a community-based population: a 10-year follow-up study. Kidney international 2007; 71: 15966.

2. Whelton PK, Carey RM, Aronow WS et al. 2017 ACC/AHA/AAPA/ABC/ACPM/AGS/APhA/ASH/ASPC/NMA/PCNA Guideline for the Prevention, Detection, Evaluation, and Management of High Blood Pressure in Adults: A Report of the American College of Cardiology/American Heart Association Task Force on Clinical Practice Guidelines. Hypertension (Dallas, Tex : 1979) 2018; 71: e13-e115.

3. Kastarinen M, Juutilainen A, Kastarinen $\mathbf{H}$ et al. Risk factors for end-stage renal disease in a community-based population: 26-year follow-up of 25,821 men and women in eastern Finland. Journal of internal medicine 2010; 267: 612-20.

4. Haroun MK, Jaar BG, Hoffman SC et al. Risk factors for chronic kidney disease: a prospective study of 23,534 men and women in Washington County, Maryland. Journal of the American Society of Nephrology : JASN 2003; 14: 2934-41.

5. Hsu CY, McCulloch CE, Darbinian J et al. Elevated blood pressure and risk of end-stage renal disease in subjects without baseline kidney disease. Archives of internal medicine 2005; 165: 923-8.

6. Tozawa $\mathrm{M}$, Iseki $\mathrm{K}$, Iseki $\mathrm{C}$ et al. Blood pressure predicts risk of developing end-stage renal disease in men and women. Hypertension (Dallas, Tex : 1979) 2003; 41: 1341-5.

7. Iseki K. Predictors of diabetic end-stage renal disease in Japan. Nephrology (Carlton, Vic) 2005; 10 Suppl: S2-6.

8. Osawa T, Fujihara K, Harada M et al. Higher pulse pressure predicts initiation of dialysis in Japanese patients with diabetes. Diabetes/metabolism research and reviews 2019; 35: e3120.

9. Kimura S, Sato T, Ikeda S et al. Development of a database of health insurance claims: standardization of disease classifications and anonymous record linkage.Journal of epidemiology 2010; 20: 413-9.

10. Fujihara K, Igarashi R, Yamamoto $M$ et al. Impact of glucose tolerance status on the development of coronary artery disease among working-age men. Diabetes $\&$ metabolism 2016. 
11. Hsieh MC, Hsieh YT, Cho TJ et al. Remission of diabetic nephropathy in type 2 diabetic Asian population: role of tight glucose and blood pressure control.European journal of clinical investigation 2011; 41: 870-8.

12. Sheen YJ, Lin JL, Li TC et al. Systolic blood pressure as a predictor of incident albuminuria and rapid renal function decline in type 2 diabetic patients.Journal of diabetes and its complications 2014; 28: 779-84.

13. Zoppini G, Targher G, Chonchol M et al. Predictors of estimated GFR decline in patients with type 2 diabetes and preserved kidney function. Clinical journal of the American Society of Nephrology : CJASN 2012; 7: 401-8.

14. Ushigome E, Hamaguchi M, Matsumoto S et al. Optimal home SBP targets for preventing the progression of diabetic nephropathy in patients with type 2 diabetes mellitus. Journal of hypertension 2015; 33: 1853-9; discussion 9.

15. Rossing K, Christensen PK, Hovind $P$ et al. Progression of nephropathy in type 2 diabetic patients. Kidney international 2004; 66: 1596-605.

16. Report of the Committee on the Classification and Diagnostic Criteria of Diabetes Mellitus: Revision for International Harmonization of HbA1c in Japan: Journal of the Japan Diabetes Society. 2012; 7: 485-504.

17. Intensive blood-glucose control with sulphonylureas or insulin compared with conventional treatment and risk of complications in patients with type 2 diabetes (UKPDS 33). UK Prospective Diabetes Study (UKPDS) Group. Lancet (London, England) 1998; 352: 837-53.

18. Nathan DM, Genuth S, Lachin $J$ et al. The effect of intensive treatment of diabetes on the development and progression of long-term complications in insulin-dependent diabetes mellitus. The New England journal of medicine 1993; 329: 977-86.

19. Ohkubo $\mathbf{Y}$, Kishikawa $\mathbf{H}$, Araki $\mathbf{E}$ et al. Intensive insulin therapy prevents the progression of diabetic microvascular complications in Japanese patients with non-insulin-dependent diabetes mellitus: a randomized prospective 6-year study. Diabetes research and clinical practice 1995; 28: 103-17.

20. Beddhu S, Greene T, Boucher $\mathrm{R}$ et al. Intensive systolic blood pressure control and incident chronic kidney disease in people with and without diabetes mellitus: secondary analyses of two randomised controlled trials. The lancet Diabetes \& endocrinology 2018; 6: 555-63.

21. Cushman WC, Evans GW, Byington RP et al. Effects of intensive blood-pressure control in type 2 diabetes mellitus. The New England journal of medicine 2010; 362: 1575-85.

22. Rahman F, McEvoy JW, Ohkuma T et al. Effects of Blood Pressure Lowering on Clinical Outcomes According to Baseline Blood Pressure and Cardiovascular Risk in Patients With Type 2 Diabetes Mellitus. Hypertension (Dallas, Tex : 1979)2019; 73: 1291-9.

23. Management of Diabetic Patients on Hemodialysis. 2012.

Table 1. Characteristics of study participants according to presence or absence of diabetes and dialysis

\begin{tabular}{|c|c|c|c|c|c|}
\hline & Total & Diabetes mellitus (-) & Diabetes mellitus (-) & $\mathrm{P}$-value & Diabetes \\
\hline & $(\mathrm{n}=258874)$ & $\begin{array}{l}\text { Dialysis } \\
(-) \\
(\mathrm{n}=241515)\end{array}$ & $\begin{array}{l}\text { Dialysis } \\
(+) \\
(\mathrm{n}=113)\end{array}$ & & $\begin{array}{l}\text { Dialysis } \\
(-) \\
(\mathrm{n}=1717\end{array}$ \\
\hline Age (years) & $45 \pm 9$ & $\mathbf{4 4} \pm 9$ & $47 \pm 8$ & ¡0.001 & $50 \pm 8$ \\
\hline Sex (Male, \%) & $161007(62)$ & $146602(61)$ & $91(81)$ & $¡ 0.001$ & 14243 \\
\hline
\end{tabular}




\begin{tabular}{llllll}
\hline & Total & Diabetes mellitus (-) & Diabetes mellitus (-) & P-value & Diabete \\
\hline Body mass index (kg/m2) & $22.8 \pm 3.6$ & $\mathbf{2 2 . 6} \pm \mathbf{3 . 4}$ & $\mathbf{2 3 . 6} \pm \mathbf{4 . 0}$ & $\mathbf{0 . 0 0 3}$ & $26.1 \pm 4$. \\
Smoking (\%) & $71904(28)$ & $\mathbf{6 5 3 6 5}(\mathbf{2 7})$ & $\mathbf{4 4}(\mathbf{3 9})$ & $\mathbf{0 . 0 0 5}$ & $6462(38$ \\
Systolic blood pressure (mmHg) & $120 \pm 16$ & $\mathbf{1 1 9} \pm \mathbf{1 5}$ & $\mathbf{1 2 9} \pm \mathbf{2 1}$ & $\mathbf{i 0 . 0 0 1}$ & $\mathbf{1 3 1} \pm \mathbf{1 7}$ \\
Diastolic blood pressure (mmHg) & $74 \pm 11$ & $\mathbf{7 3} \pm \mathbf{1 1}$ & $\mathbf{7 9} \pm \mathbf{1 5}$ & $\mathbf{i} \mathbf{0 . 0 0 1}$ & $80 \pm 11$ \\
HbA1c (\%) & $5.5 \pm 0.7$ & $5.4 \pm 0.3$ & $5.5 \pm 0.4$ & 0.243 & $\mathbf{7 . 2} \pm \mathbf{1 . 4}$ \\
LDL cholesterol (mmol/L) & $3.1 \pm 0.8$ & $3.1 \pm 0.8$ & $3.0 \pm 0.9$ & 0.470 & $3.3 \pm 0.9$ \\
HDL cholesterol (mmol/L) & $1.6 \pm 0.4$ & $\mathbf{1 . 7} \pm \mathbf{0 . 4}$ & $\mathbf{1 . 4} \pm \mathbf{0 . 4}$ & $\mathbf{i 0 . 0 0 1}$ & $\mathbf{1 . 4} \pm \mathbf{0 . 4}$ \\
Medication for diabetes (\%) & $8136(3)$ & - & - & & $\mathbf{8 0 8 0}(4)$ \\
Medication for hypertension (\%) & $21103(8)$ & $\mathbf{1 5 7 7 7}(\mathbf{7})$ & $\mathbf{5 1}(\mathbf{4 5})$ & $\mathbf{i 0 . 0 0 1}$ & $\mathbf{5 2 1 8}(\mathbf{3}$ \\
Prevalence of coronary artery disease (\%) & $13055(5)$ & $\mathbf{1 0 6 0 1}(\mathbf{4})$ & $\mathbf{3 7}(\mathbf{3 3})$ & $\mathbf{i 0 . 0 0 1}$ & $\mathbf{2 3 8 2}(1)$ \\
\hline
\end{tabular}

HbA1c: glycated hemoglobin A1c; LDL cholesterol: low-density lipoprotein cholesterol; HDL cholesterol: high-density lipoprotein cholesterol

Data are presented as numbers, means \pm standard deviations, or percentages.

Table 2. Cox regression analysis of variables for the incidence of dialysis in participants with and without diabetes mellitus (DM)

\begin{tabular}{|c|c|c|c|c|c|c|c|c|c|c|c|c|c|}
\hline \multicolumn{3}{|c|}{ Total } & $\begin{array}{l}\mathrm{DM} \\
(-)\end{array}$ & $\begin{array}{l}\text { DM } \\
(-)\end{array}$ & $\begin{array}{l}\text { DM } \\
(-)\end{array}$ & $\begin{array}{l}\text { DM } \\
(-)\end{array}$ & $\begin{array}{l}\text { DM } \\
(-)\end{array}$ & $\begin{array}{l}\text { DM } \\
(-)\end{array}$ & $\begin{array}{l}\text { DM } \\
(-)\end{array}$ & $\begin{array}{l}\text { DM } \\
(-)\end{array}$ & $\begin{array}{l}\text { DM } \\
(-)\end{array}$ & $\begin{array}{l}\text { DM } \\
(-)\end{array}$ & $\begin{array}{l}\text { DM } \\
(-)\end{array}$ \\
\hline \multicolumn{3}{|c|}{ Model } & Model & & Model & & Model & & Model & & Mode & & Mo \\
\hline \multicolumn{3}{|c|}{1} & $2 \mathrm{a}$ & & $2 \mathrm{~b}$ & & $2 \mathrm{c}$ & & $2 \mathrm{~d}$ & & $2 \mathrm{e}$ & & $2 \mathrm{f}$ \\
\hline & $\mathrm{HR}$ & (95\%R & $(95 \% \mathrm{dR}$ & (95\%R & (95\%R & (95 & (95\%R & (95 & (95\%R & (95\%R & (95\%R & (95\%R & $(95 \% R$ \\
\hline & CI) & CI) & CI) & CI) & CI) & CI) & CI) & CI) & CI) & CI) & CI) & CI) & CI) \\
\hline & $\begin{array}{l}\mathrm{HR} \\
(05 \%\end{array}$ & HR & $\begin{array}{l}\mathrm{HR} \\
(05 \%\end{array}$ & $\begin{array}{l}\mathrm{HR} \\
05 \% \%\end{array}$ & $\begin{array}{l}\mathrm{HR} \\
(95 \%\end{array}$ & $\begin{array}{l}\mathrm{HR} \\
(95 \%\end{array}$ & $\begin{array}{l}\mathrm{HR} \\
(9.5 \%\end{array}$ & $\begin{array}{l}\mathrm{HR} \\
(95 \%\end{array}$ & $\begin{array}{l}\mathrm{HR} \\
(9.5 \%\end{array}$ & $\begin{array}{l}\mathrm{HR} \\
(95 \%\end{array}$ & $\begin{array}{l}\mathrm{HR} \\
(95 \%\end{array}$ & $\begin{array}{l}\mathrm{HR} \\
(95 \%\end{array}$ & $\begin{array}{l}\mathrm{HR} \\
\left(95^{\circ}\right.\end{array}$ \\
\hline & $\begin{array}{l}(95 \% \\
\mathrm{CI})\end{array}$ & $\begin{array}{l}(95 \% \\
\text { CI) }\end{array}$ & $\begin{array}{l}(95 \% \\
\mathrm{CI})\end{array}$ & $\begin{array}{l}(95 \% \\
\text { CI })\end{array}$ & $\begin{array}{l}(95 \% \\
\text { CI })\end{array}$ & $\begin{array}{l}(95 \% \\
\text { CI })\end{array}$ & $\begin{array}{l}(95 \% \\
\mathrm{CI})\end{array}$ & $\begin{array}{l}(95 \% \\
\text { CI })\end{array}$ & $\begin{array}{l}(95 \% \\
\mathrm{CI})\end{array}$ & $\begin{array}{l}(95 \% \\
\text { CI })\end{array}$ & $\begin{array}{l}(95 \% \\
\text { CI) }\end{array}$ & $\begin{array}{l}(95 \% \\
\text { CI) }\end{array}$ & $\begin{array}{l}(95 \% \\
\text { CI })\end{array}$ \\
\hline & $\begin{array}{l}\text { HR } \\
(95 \%\end{array}$ & $\begin{array}{l}\mathrm{HR} \\
(95 \%\end{array}$ & $\begin{array}{l}\mathrm{HR} \\
(95 \%\end{array}$ & $\begin{array}{l}\mathrm{HR} \\
(95 \%\end{array}$ & $\begin{array}{l}\mathrm{HR} \\
(95 \%\end{array}$ & $\begin{array}{l}\mathrm{HR} \\
(95 \%\end{array}$ & $\begin{array}{l}\mathrm{HR} \\
(95 \%\end{array}$ & $\begin{array}{l}\mathrm{HR} \\
(95 \%\end{array}$ & $\begin{array}{l}\mathrm{HR} \\
(95 \%\end{array}$ & $\begin{array}{l}\mathrm{HR} \\
(95 \%\end{array}$ & $\begin{array}{l}\mathrm{HR} \\
(95 \%\end{array}$ & $\begin{array}{l}\mathrm{HR} \\
(95 \%\end{array}$ & $\mathrm{HR}$ \\
\hline & $\begin{array}{l}\text { CI) } \\
\text { (95\% }\end{array}$ & $\begin{array}{l}(95 \% \\
\mathrm{CI})\end{array}$ & $\begin{array}{l}\text { CI) } \\
\text { (95\% }\end{array}$ & $\begin{array}{l}(95 \% \\
\mathrm{CI})\end{array}$ & $\begin{array}{l}\text { CI) } \\
\text { (95\% }\end{array}$ & $\begin{array}{l}\text { CI) } \\
\text { (I) }\end{array}$ & $\begin{array}{l}\text { CI) } \\
\text { (95\% }\end{array}$ & $\begin{array}{l}\text { CI) } \\
\text { (1) }\end{array}$ & $\begin{array}{l}\text { CI) } \\
\text { (95\% }\end{array}$ & $\begin{array}{l}\text { CI) } \\
\text { CI\% }\end{array}$ & $\begin{array}{l}\text { CI) } \\
\text { CI\% }\end{array}$ & $\begin{array}{l}\text { CI) } \\
\text { (15\% }\end{array}$ & $\begin{array}{l}(959 \\
\text { CI) }\end{array}$ \\
\hline & $\mathrm{HR}$ & $\mathrm{HR}$ & $\mathrm{HR}$ & $\mathrm{HR}$ & $\mathrm{HR}$ & $\mathrm{HR}$ & $\mathrm{HR}$ & $\mathrm{HR}$ & $\mathrm{HR}$ & $\mathrm{HR}$ & $\mathrm{HR}$ & $\mathrm{HR}$ & $\mathrm{HR}$ \\
\hline & $(95 \%$ & $(95 \%$ & $(95 \%$ & $(95 \%$ & $(95 \%$ & $(95 \%$ & $(95 \%$ & $(95 \%$ & $(95 \%$ & $(95 \%$ & $(95 \%$ & $(95 \%$ & \\
\hline & CI) & CI) & CI) & CI) & CI) & CI) & CI) & CI) & CI) & CI) & CI) & CI) & CI) \\
\hline & HR & HR & $\mathrm{HR}$ & $\mathrm{HR}$ & HR & $\mathrm{HR}$ & $\mathrm{HR}$ & $\mathrm{HR}$ & $\mathrm{HR}$ & $\mathrm{HR}$ & HR & $\mathrm{HR}$ & HR \\
\hline & $(95 \%$ & $(95 \%$ & $(95 \%$ & $(95 \%$ & $(95 \%$ & $(95 \%$ & $(95 \%$ & $(95 \%$ & $(95 \%$ & $(95 \%$ & $(95 \%$ & $(95 \%$ & \\
\hline & CI) & CI) & CI) & CI) & CI) & CI) & CI) & CI) & CI) & CI) & CI) & CI) & CI) \\
\hline & $\mathrm{HR}$ & $\mathrm{HR}$ & HR & HR & $\mathrm{HR}$ & HR & $\mathrm{HR}$ & $\mathrm{HR}$ & $\mathrm{HR}$ & $\mathrm{HR}$ & $\mathrm{HR}$ & $\mathrm{HR}$ & $\mathrm{HR}$ \\
\hline & $(95 \%$ & $(95 \%$ & $(95 \%$ & $(95 \%$ & $(95 \%$ & $(95 \%$ & $(95 \%$ & $(95 \%$ & $(95 \%$ & $(95 \%$ & $(95 \%$ & $(95 \%$ & \\
\hline & CI) & CI) & CI) & CI) & CI) & CI) & CI) & CI) & CI) & CI) & CI) & CI) & CI) \\
\hline & HR & $\mathrm{HR}$ & $\mathrm{HR}$ & $\mathrm{HR}$ & $\mathrm{HR}$ & HR & HR & $\mathrm{HR}$ & $\mathrm{HR}$ & $\mathrm{HR}$ & HR & HR & $\mathrm{HR}$ \\
\hline & $(95 \%$ & $(95 \%$ & $(95 \%$ & $(95 \%$ & $(95 \%$ & $(95 \%$ & $(95 \%$ & $(95 \%$ & $(95 \%$ & $(95 \%$ & $(95 \%$ & $(95 \%$ & \\
\hline & CI) & CI) & CI) & CI) & CI) & CI) & CI) & CI) & CI) & CI) & CI) & CI) & CI) \\
\hline & $\mathrm{HR}$ & $\mathrm{HR}$ & $\mathrm{HR}$ & $\mathrm{HR}$ & $\mathrm{HR}$ & $\mathrm{HR}$ & $\mathrm{HR}$ & $\mathrm{HR}$ & $\mathrm{HR}$ & $\mathrm{HR}$ & $\mathrm{HR}$ & $\mathrm{HR}$ & HR \\
\hline & $(95 \%$ & $(95 \%$ & $(95 \%$ & $(95 \%$ & (95\% & $(95 \%$ & $(95 \%$ & $(95 \%$ & $(95 \%$ & (95\% & (95\% & (95\% & \\
\hline & CI) & CI) & CI) & CI) & CI) & CI) & CI) & CI) & CI) & CI) & CI) & CI) & CI \\
\hline Diabetes & $\begin{array}{l}3.41 \\
(2.45 \\
4.76)\end{array}$ & & - & & & & . & & & & & & \\
\hline
\end{tabular}




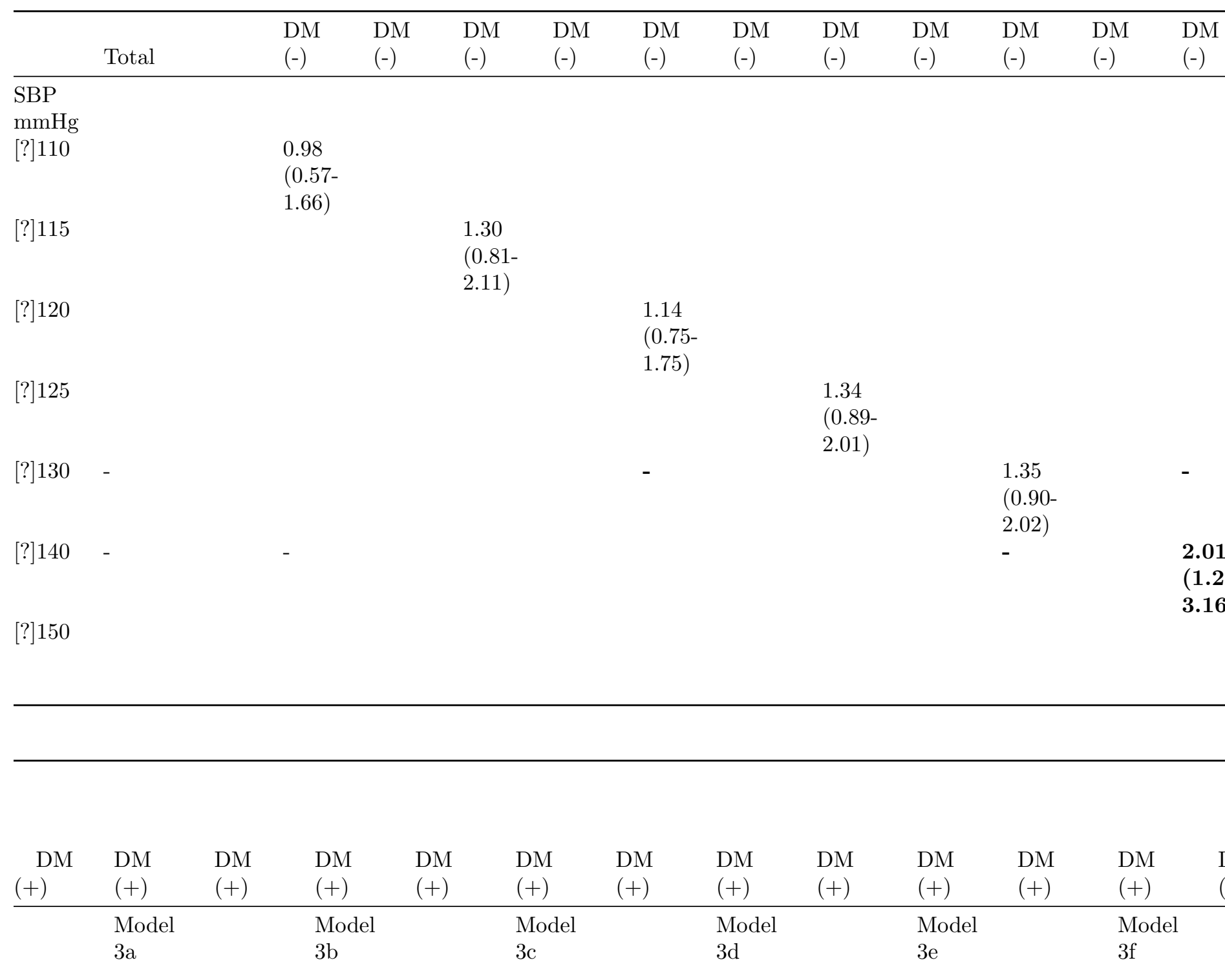




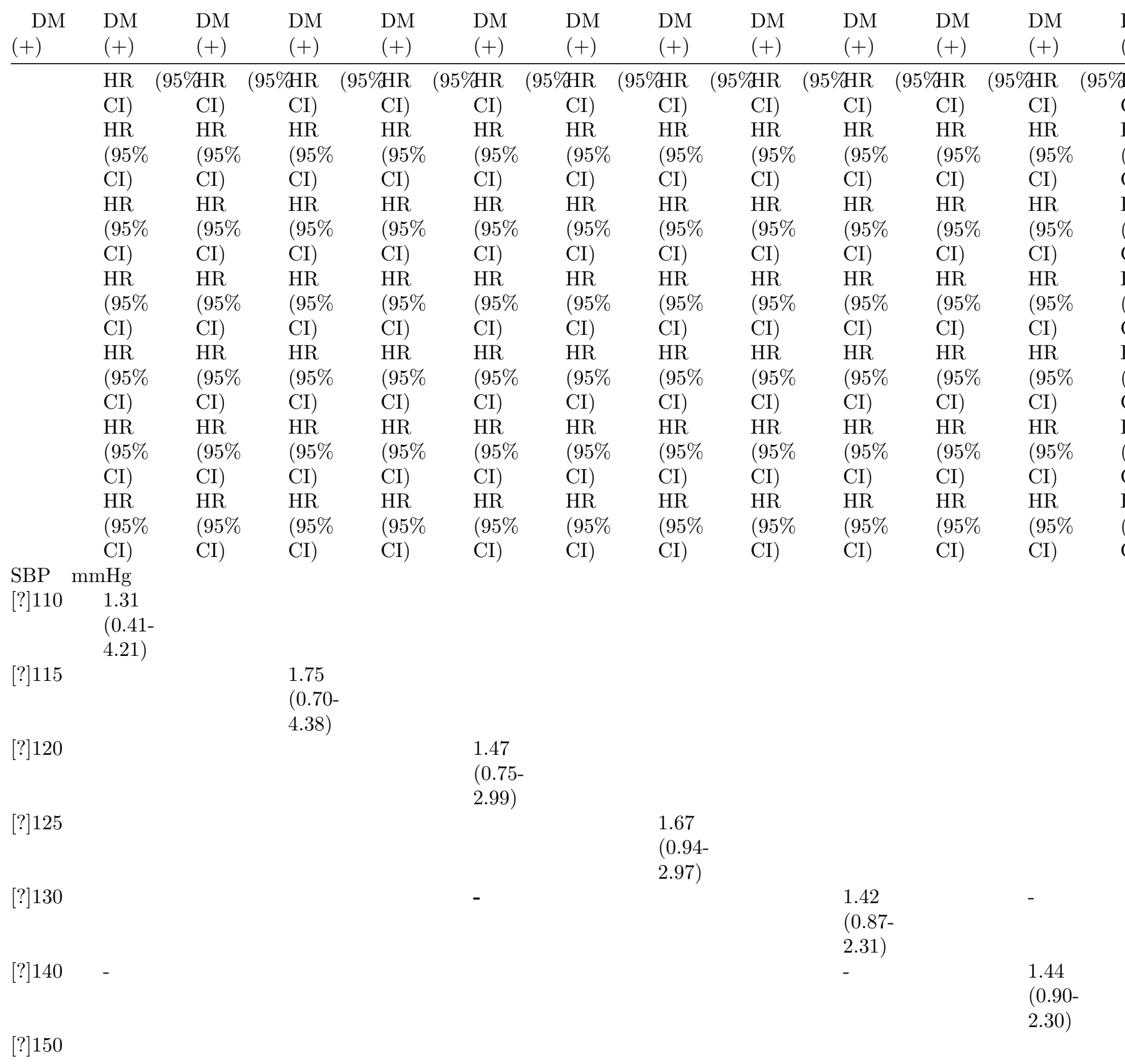

Baseline variables for predictors of dialysis adjusted by age, sex, smoking, medication for hypertension, BMI, LDL-C, HDL-C. 
HR: hazard ratio; SBP: systolic blood pressure; BMI: body mass index; LDL-C: low-density lipoprotein cholesterol; HDL-C: high-density lipoprotein cholesterol

(Model 1) Adjusted for age, sex, smoking, medication for hypertension, BMI, LDL-C, HDL-C, and DM; (Model 2-3) Adjusted for age, sex, smoking, medication for hypertension, BMI, LDL-C, HDL-C, SBP ([?]110 or [?]115 or [?]120 or [?]125 or [?]130 or [?]140 or [?]150 mmHg)

Table 3. HRs for initiation of dialysis according to combinations of DM and SBP

\begin{tabular}{|c|c|c|}
\hline $\mathrm{SBP}$ & $\mathrm{DM}(-)$ & $\mathrm{DM}(+)$ \\
\hline & $\operatorname{HR}(95 \% \mathrm{CI})$ & HR $(95 \%$ CI $) ? ¿ ?$ \\
\hline $119 \mathrm{mmHg}$ & 1.00 (Ref.) & 1.00 (Ref.) \\
\hline $120-129 \mathrm{mmHg}$ & $0.98(0.58-1.64)$ & $1.22(0.55-2.70)$ \\
\hline $130-139 \mathrm{mmHg}$ & $0.94(0.52-1.67)$ & $1.41(0.65-3.04)$ \\
\hline $140-149 \mathrm{mmHg}$ & $1.28(0.63-2.61)$ & $1.31(0.55-3.14) ? \gtreqless ?$ \\
\hline $150 \mathrm{mmHg}$ & $2.87(1.55-5.32)$ & $2.28(1.03-5.01) ? ¿ ?$ \\
\hline $119 \mathrm{mmHg}$ & 1.00 (Ref.) & $3.01(1.45-6.25)$ \\
\hline 120-129 mmHg & $0.97(0.58-1.62)$ & $3.70(1.96-7.00)$ \\
\hline 130-139 mmHg & $0.95(0.54-1.68)$ & $4.26(2.32-7.81)$ \\
\hline $140-149 \mathrm{mmHg}$ & $1.31(0.65-2.65)$ & $3.94(1.89-8.19) ? \gtreqless ?$ \\
\hline $150 \mathrm{mmHg}$ & $3.00(1.65-5.44)$ & $6.88(3.66-12.9)$ \\
\hline
\end{tabular}

HR for initiation of dialysis compared with the combination of DM (-) and SBP [?]119 mmHg /DM (+) and SBP [?]119 $\mathrm{mmHg}$ as a reference group. Baseline variables as predictors for dialysis adjusted by age, sex, smoking, medication for hypertension, BMI, LDL-C, HDL-C.

HR: hazard ratio; DM: diabetes mellitus; SBP: systolic blood pressure; BMI: body mass index; LDL-C: low-density lipoprotein cholesterol; HDL-C: high-density lipoprotein cholesterol

Figure legends

Figure 1 Kaplan-Meier analysis of unadjusted overall time to initiation of dialysis

Kaplan-Meier analysis of unadjusted overall time to initiation of dialysis for 5 groups without DM according to SBP ([?]119 or 120-129 or 130-139 or 140-149 or [?]150 mmHg). (B) Kaplan-Meier analysis of unadjusted overall time to initiation of dialysis for 5 groups with DM according to SBP ([?]119 or 120-129 or 130-139 or 140-149 or [?]150 mmHg). DM: diabetes mellitus: SBP: systolic blood pressure 

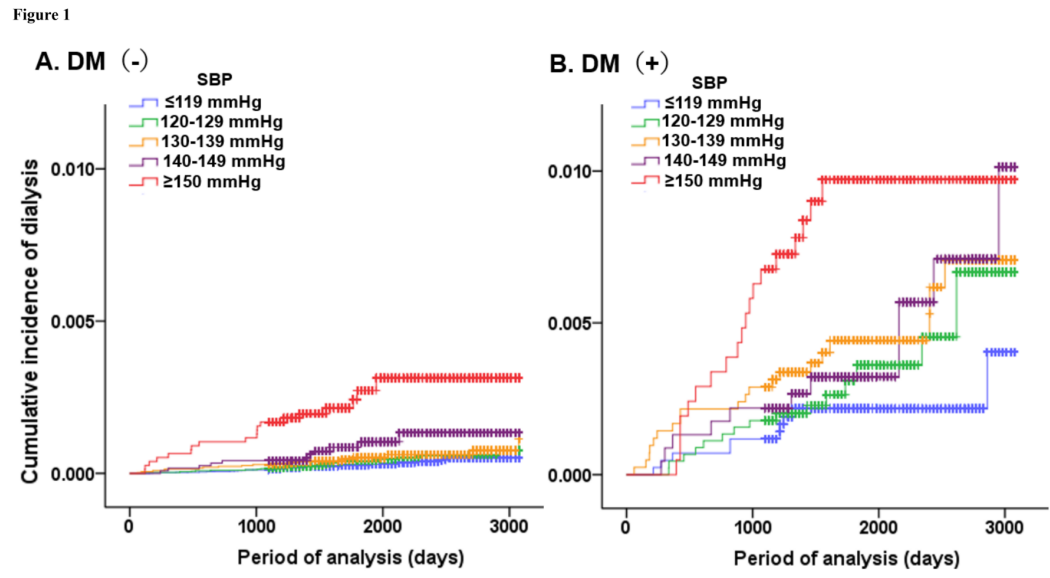